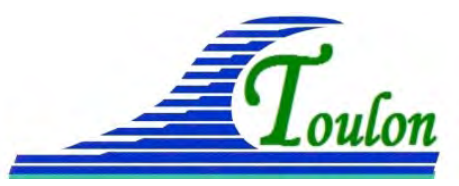

XIV $V^{\text {èmes }}$ Journées Nationales Génie Côtier - Génie Civil

Toulon, 29 juin au $1^{\text {er }}$ juillet 2016

DOI:10.5150/jngcgc.2016.019 @ Editions Paralia CFL

disponible en ligne - http://www.paralia.fr - available online

\title{
Réponse d'une plage sableuse aux événements de tempête : variabilité longshore et impacts d'ouvrages
}

\author{
Mélanie BIAUSQUE ${ }^{1}$, Nadia SENECHAL ${ }^{1}$, Adeline BARRE ${ }^{1}$
}

\author{
1. Université de Bordeaux, UFR Sciences et Techniques, UMR CNRS 5805 EPOC, \\ Allée Geoffroy Saint-Hilaire, CS 50023, 33615 Pessac Cedex, France. \\ melanie.biausque@u-bordeaux.fr ; nadia.senechal@u-bordeaux.fr ; \\ adeline.barre@etu.u-bordeaux.fr
}

\section{Résumé :}

La Côte Aquitaine est un littoral sableux linéaire ouvert sur les houles océaniques attirant de multiples activités socio-économiques (notamment le tourisme). Comme la plupart des plages d'Aquitaine, la plage de Biscarrosse est une plage ouverte dominée par la houle. Elle présente l'avantage de regrouper plusieurs modes de gestion : la partie Nord de la plage de Biscarrosse est très faiblement anthropisée par des brise-vent déployés par l’ONF sur la dune, la partie Sud de Biscarrosse Plage, au contraire, est fortement anthropisée avec la présence d'enrochements. Des levés DGPS haute fréquence ont été effectués durant le 1er cluster de tempêtes de l'hiver 2015/2016 dans le but de caractériser l'évolution morphologique de la plage de Biscarrosse. A partir de ces levés, des profils topographiques ont pu être extraits avant et après chaque tempête permettant d'évaluer s’il existe une variabilité longitudinale dans la réponse de la plage. Les premiers résultats montrent que la partie Sud semble être plus impactée par la succession de tempêtes qui ont eu lieu en Janvier 2016. Lors de la 3ème tempête du cluster, une falaise d'érosion d'environ $2 \mathrm{~m}$ de hauteur s'est créée de part et d'autre des enrochements, provoquée par une perte nette de sédiment du haut de plage et associée à un recul du pied de dune de plus de $15 \mathrm{~m}$. Les profils topographiques du Nord de la plage, quant à eux, témoignent d'un rééquilibrage progressif de celle-ci vers un profil de type hivernal, ainsi que d'un léger recul du pied de dune. Cette variabilité longshore est vraisemblablement due, en partie, à la présence d'enrochements. Leur impact a pu être mis en relation avec de précédentes études, proposant une "érosion active" liée à la réflexion des vagues de long de l'ouvrage. Néanmoins, un courant d'arrachement a été observé au niveau des ouvrages du Sud de la plage. Une future analyse d’images vidéo pourrait expliquer le rôle de ce courant dans l'évolution du système.

Mots-clés : Morphodynamique des plages, Dynamique sédimentaire, Profils topographiques, Cluster de tempête, Impacts d’ouvrages

\section{Introduction}

Le taux de population vivant près du littoral ne cesse d'augmenter impliquant des pressions anthropiques de plus en plus marquées, notamment liées au tourisme. Mais les 


\section{Thème 2 - Dynamique sédimentaire}

structures construites le long des côtes sableuses, et les activités humaines qui en découlent, se voient menacées par la mobilité du trait de côte. D'après PILKEY et HUME (2001), 80\% du trait de côte mondial est en recul, associé à un abaissement du niveau des plages. En réponse à ces perturbations, les gestionnaires mettent en place des aménagements littoraux afin de préserver des zones naturelles comme les dunes, mais également de conserver une largeur de plage suffisante pour assurer un bon rendement touristique, ou encore de maintenir en place les constructions littorales. Après étude des sites et des enjeux (économiques, scientifiques et sociaux) deux types d'aménagement sont préférentiellement choisis pour stabiliser le trait de côte (MICALLEF \& WILLIAMS, 2002): (1) les constructions en dur, comme les digues ou les enrochements, ont pour vocation de fixer le trait de côte en un endroit donné ; (2) les stabilisations "douces", comme le ré-ensablement des plages, présentent un rôle différent, elles permettent de créer une zone 'tampon' comme par exemple lors des évènements de tempête. Même si les constructions en dur semblent plus définitives, chaque type d'aménagement impacte le système dune/plage à différentes échelles de temps (ELLS \& MURRAY, 2012). Avec ses $250 \mathrm{~km}$ de littoral linéaire, la côte Aquitaine n'est pas épargnée par les problèmes d'érosion du trait de côte et de gestion de cette érosion. Durant l'hiver 2013/2014, les impacts des successions de tempêtes sur la plage, le cordon dunaire et les activités socio-économiques associées ont été considérables (CASTELLE et al., 2015). Les communes accordent donc une importance accrue à l'étude des tempêtes hivernales afin d'apporter des réponses concrètes concernant la lutte contre l'érosion du littoral, ainsi que les possibilités d'aménagement et leurs impacts. Dans ce but, des levés topographiques haute fréquence ont été réalisés sur la plage de Biscarrosse (Aquitaine-France) pendant la première succession de tempêtes de l’hiver 2015/2016.

\section{Matériels et méthodes}

\subsection{Zone d'étude}

Le site choisi pour cette étude est la plage de Biscarrosse, située sur la côte Aquitaine dans le Sud-Ouest de la France (fig. 1). Cette plage est décrite comme typique de la morphologie caractéristique des plages d'Aquitaine (e.g. CASTELLE et al., 2007). Avec une orientation de $280,5^{\circ} \mathrm{N}$, cette plage est totalement ouverte à la houle nord Atlantique. Les hauteurs significatives ( $H s$ ) moyennes annuelles sont de $1,4 \mathrm{~m}$ et associées à des périodes pic (Tp) de 6,5 s (BUTEL et al., 2002). Néanmoins, le régime des vagues est soumis à une forte variabilité saisonnière, avec des hauteurs maximales pouvant dépasser les $10 \mathrm{~m}$ en hiver (Novembre à Mars). Le marnage est de type meso à macro-tidal, avec des valeurs autour de 3,7 $\mathrm{m}$ en vives-eaux et 1,8 $\mathrm{m}$ en mortes-eaux.

Alimentée par la dérive littorale d'orientation nord-sud, la plage de Biscarrosse se compose d'un sédiment moyen dont la taille médiane des grains est de 0,35 mm (BA \& 


\section{XIV ${ }^{\text {èmes }}$ Journées Nationales Génie Côtier - Génie Civil \\ Toulon, 29 juin au $1^{\text {er }}$ juillet 2016}

SENECHAL, 2013). Selon la classification WRIGHT et SHORT (1984), cette plage est dite "intermédiaire" et les états TBR (Transverse Bar and Rip) à LTT (Low Tide Terrace) sont les plus fréquemment observés (PERON \& SENECHAL, 2011). La plage de Biscarrosse est représentative d'un système de double barre (ALMAR et al., 2009). Alors que la barre interne présente des structures complexes en 3D (PERON \& SENECHAL, 2011), la barre externe est généralement en forme de croissants (e.g. CASTELLE et al., 2007).

En matière de gestion et d'aménagements, Biscarrosse est d'un grand intérêt puisque sa partie sud est fortement anthropisée par la mise en place de protections de type enrochements, alors que la partie nord n'est gérée que par des brise-vent de type ganivelles déployés sur la dune (fig.1).

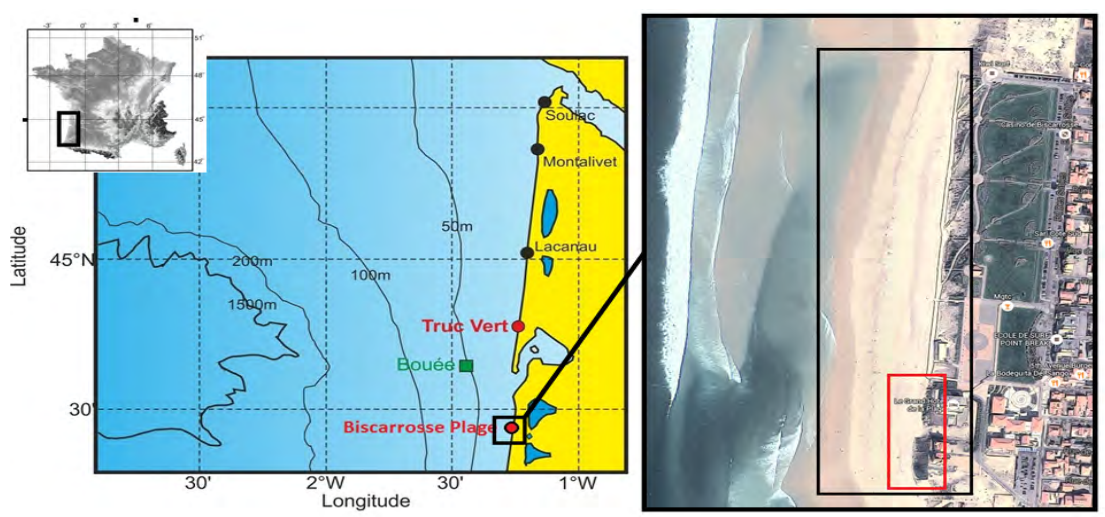

Figure 1. Localisation de la plage de Biscarrosse. A droite: le rectangle noir représente la zone complète d'étude de levés topographiques, le rectangle rouge représente la zone d'enrochements.

\subsection{Données environnementales}

Les données de houle, présentées dans la figure 2, ont été extraites de la base de données nationale de mesures CANDHIS. La campagne effectuée au large du CapFerret (Gironde) résulte de la collaboration entre le CEREMA, l’Université de Bordeaux et le SHOM.

\subsection{Données in-situ}

Pour caractériser l'évolution morphodynamique de la plage, un suivi topographique haute fréquence est effectué durant le $1^{\text {er }}$ cluster de tempêtes de l'hiver 2015-2016. Entre les 05 et 13 Janvier, des levés au GPS différentiel (DGPS) sont acquis quotidiennement, à marée basse, puis tous les deux jours entre les 15 et 19 Janvier ; soient 12 levés au total. Sur environ $700 \mathrm{~m}$ de longueur de plage, 31 transects crossshore, séparés d’une 20aine de mètres chacun, sont enregistrés, couvrant la totalité de la plage et une partie de la dune. Le but de cette étude étant d'identifier s'il existe une 


\section{Thème 2 - Dynamique sédimentaire}

disparité longitudinale dans la réponse de la plage aux évènements de tempête, des profils sont extraits en trois positions différentes le long de la plage. Ainsi, les cas couvrent les deux modes de gestion employés à Biscarrosse : le profil C caractérise la partie extrême sud de la zone d'étude, définie par la présence d'enrochements, le profil B se situe à la limite nord des enrochements, alors que le profil A représente la zone qui n'est soumise à aucune construction massive de stabilisation.

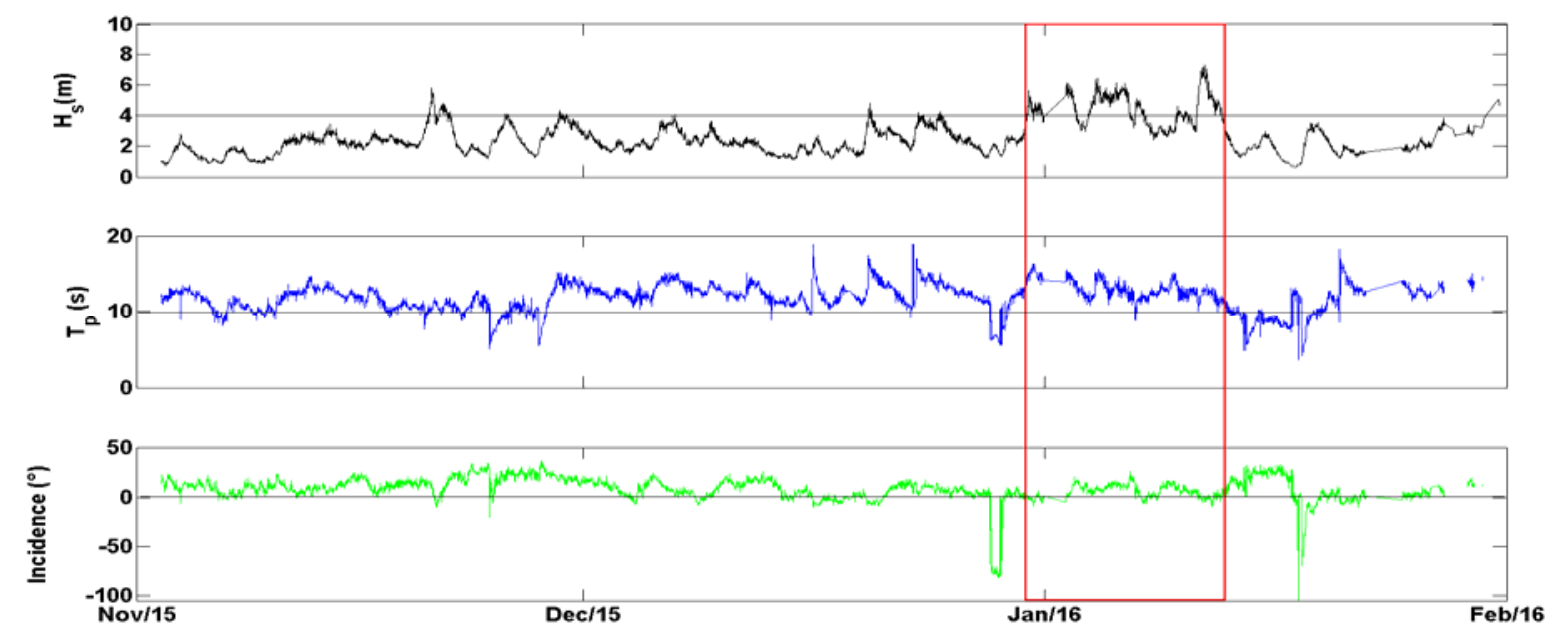

Figure 2. Données de houle. En noir les hauteurs significatives des vagues (Hs, en mètres), en bleu les périodes pic (Tp, en secondes) et en vert l'incidence des vagues (en degrés), en rouge le $1^{\text {er }}$ cluster de l'hiver.

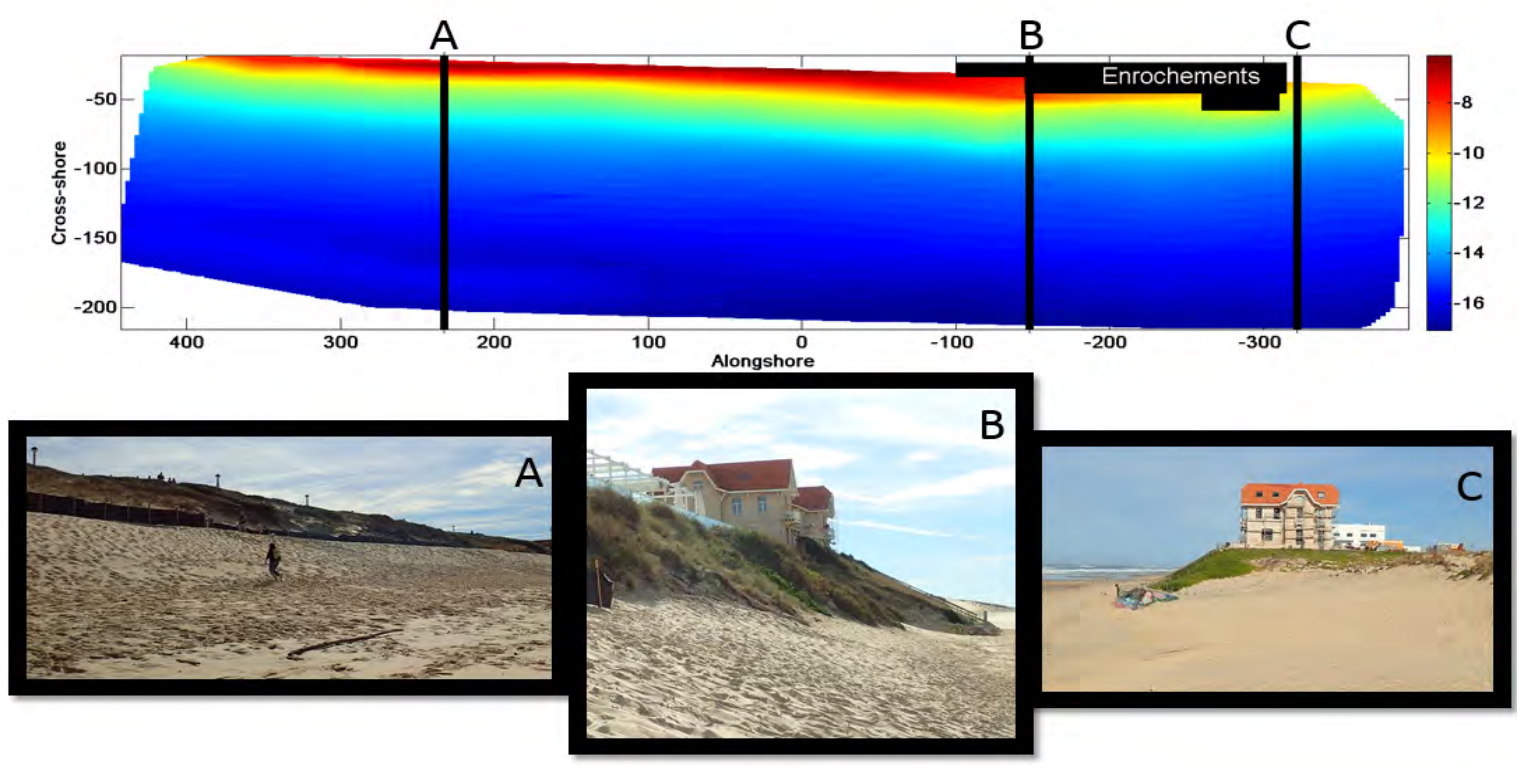

Figure 3. Localisation des profils sur un exemple de carte topographique construite à partir des levés DGPS. 


\section{XIV ${ }^{\text {èmes }}$ Journées Nationales Génie Côtier - Génie Civil \\ Toulon, 29 juin au $1^{\text {er }}$ juillet 2016}

\section{Résultats et discussions}

\subsection{Conditions hydrodynamiques}

En moyenne, entre novembre 2015 et février 2016, la hauteur significative (Hs) des vagues est de 2,5 m pour des périodes pic ( $T p$ ) de l'ordre de $10 \mathrm{~s}$ (fig. 2). Le Hs maximal atteint néanmoins les 7,32 m associé une période de 13,1 s le 11 Janvier 2016. Concernant l'incidence des vagues, la houle est principalement de secteur Nord-Ouest. Selon la définition proposée par DOLAN et DAVIS (1994), un climat de tempête se caractérise par des hauteurs de vagues significatives dépassant un seuil donné pendant une durée de temps minimale. Le climat de tempête hivernal pour la plage de Biscarrosse a été déterminé par des $H s>4 \mathrm{~m}$ (soit généralement moins de $2 \%$ des vagues en hiver) pendant un cycle de marée complet, soit 12h (SENECHAL et al., 2015). Grâce à ces critères, trois tempêtes ont pu été identifiées : la première tempête, datant du 2 Janvier 2016, s’est déroulée pendant une marée de mortes-eaux (coefficient de marée égal à 40) sur une période de 20 h, avec des hauteurs de vagues moyennes de $5,2 \mathrm{~m}$, et une hauteur maximale de 6,1 m. La seconde s'est étendue sur plus de $90 \mathrm{~h}$ allant du 4 au 7 Janvier. Les vagues ont atteint jusqu'à 6,5 m de hauteur significative avec une moyenne $5 \mathrm{~m}$. La dernière tempête, datant des 11 et 12 Janvier, se démarque des deux autres d'un point de vue hydrodynamique puisqu'elle s'est déroulée pendant une période de vives-eaux (coefficient à 97). Elle a duré environ $40 \mathrm{~h}$, avec des $H s$ allant jusqu’à 7,32 m pour des périodes pic de 13,1 s, et un $H s$ moyen de 5,5 m. Compte tenu du faible intervalle de temps entre les évènements (< 3-4 jours), ces évènements peuvent être considérés comme formant un cluster.

\subsection{Réponses de la plage aux conditions de tempêtes}

Une variation longitudinale de la réponse du système dune/plage a pu être observée insitu (fig. 4).

Les observations de terrains sont confirmées par les données DGPS et les profils topographiques cross-shore (fig.5 à 7). Afin de couvrir au mieux le cluster et d'identifier l'impact de chaque tempête, les profils ont été extraits avant et après chaque évènement. Ainsi les figures 5 à 7 représentent l'évolution morphologique de la plage en trois positions.

La partie nord de la plage a été érodée progressivement par la succession d'évènements énergétiques (fig.5). Le profil du 24 Novembre est le profil de base de la plage, avant l'arrivée de la première tempête. Comme vu dans la partie précédente, la $1^{\text {ère }}$ tempête du cluster s'est déroulée le 2 Janvier. La campagne de terrain n’ayant commencée qu’à partir du 5 Janvier, il est impossible d'évaluer avec précision l'importance de la première tempête sur la plage. Néanmoins, le profil du 5 Janvier montre un maintien du pied de dune ainsi qu'un léger engraissement du haut de plage. Le profil rouge (8 Janvier) donne la position de la plage après le passage de la seconde tempête. On 


\section{Thème 2 - Dynamique sédimentaire}

observe une érosion du haut et du bas de plage, la dune n'étant pas impactée. Le profil du 13 Janvier montre la topographie après le dernier évènement du cluster. Une importante érosion du haut de plage (environ $50 \mathrm{~cm}$ ) est notable et s'associe à une accentuation de la pente de la plage liée à un recul du pied de dune de $6 \mathrm{~m}$. Le profil du 19 Janvier confirme la nouvelle position du trait de côte, avec un début de réengraissement du bas de plage.
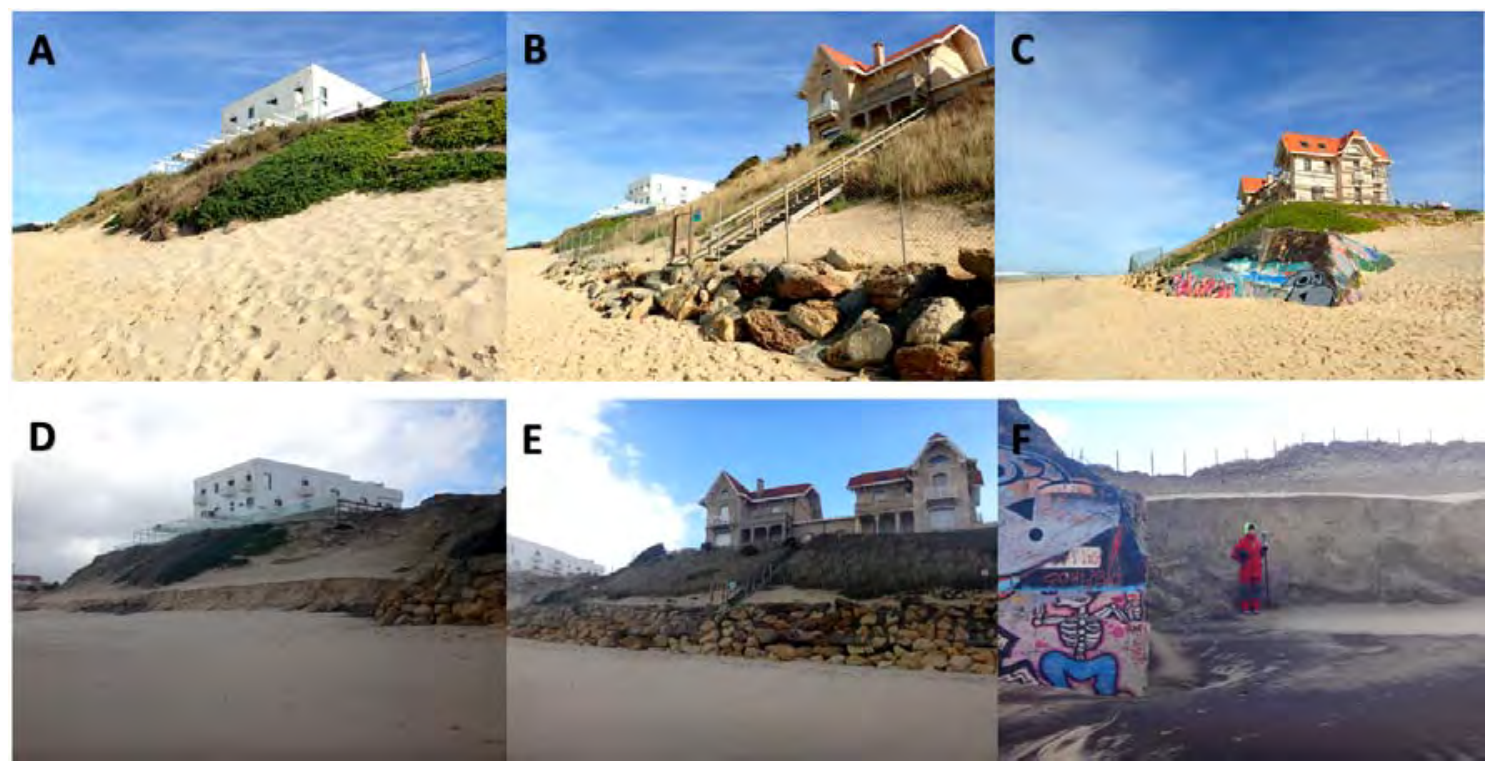

Figure 4. Situation de part et d'autre des enrochements avant $(A, B, C)$ et après $(D, E$, F) le cluster.

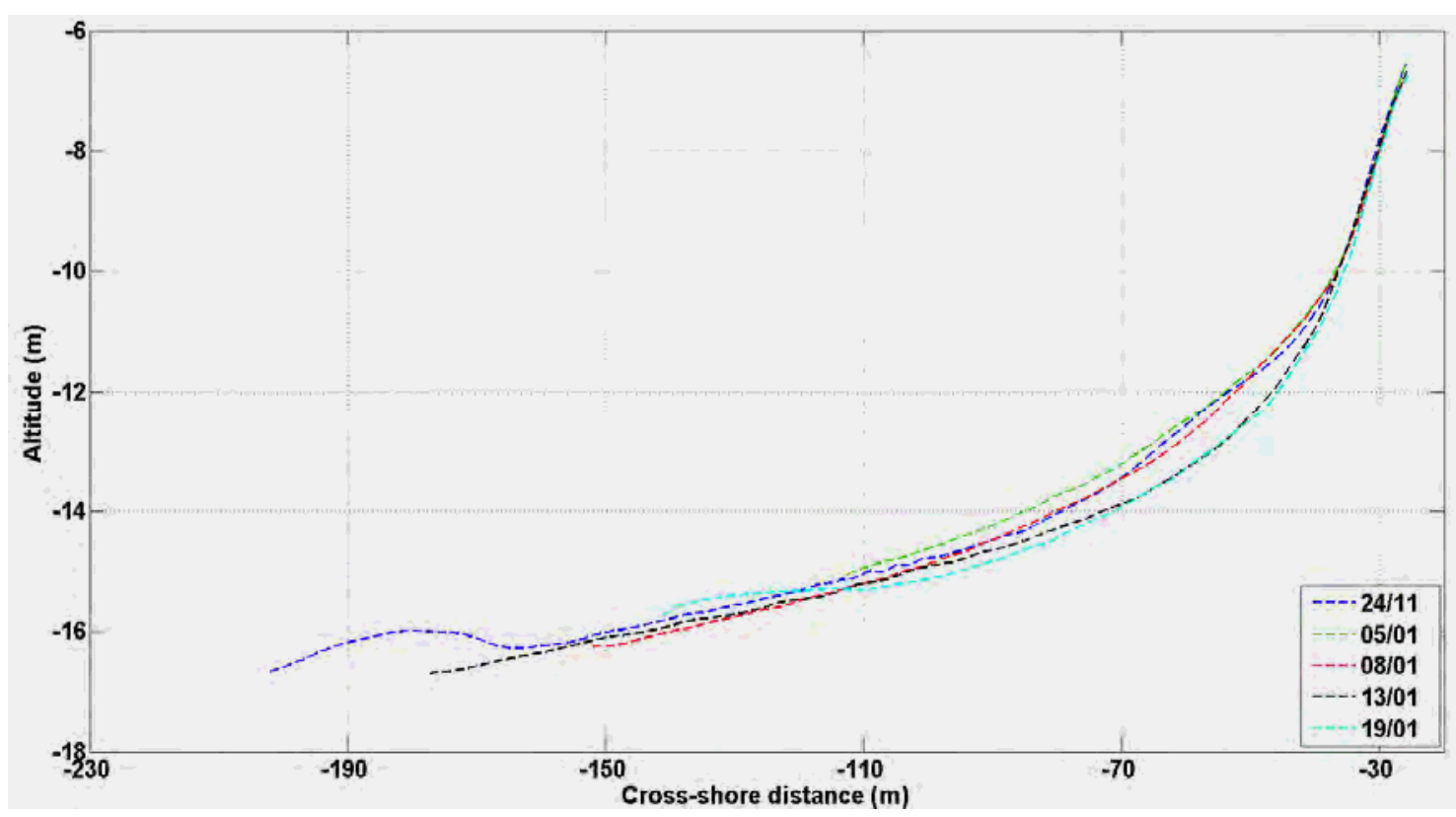

Figure 5. Profils topographiques représentant la partie nord de la plage, Profil A (non anthropisée). Les données sont exprimées en valeurs absolues. 


\section{XIV ${ }^{\text {èmes }}$ Journées Nationales Génie Côtier - Génie Civil \\ Toulon, 29 juin au $1^{\text {er }}$ juillet 2016}

L’érosion subie par partie sud de la plage a été bien plus marquée que dans la partie nord. En effet, après la seconde tempête (profil du 8 Janvier) le haut de plage connaît une érosion de plus de $50 \mathrm{~cm}$, raidissant la pente de plage, que ce soit au nord ou au sud des enrochements (fig. 6 et 7). Par contre, l'impact de la dernière tempête a été plus problématique dans la zone au sud des constructions de stabilisation. Une falaise d'érosion de $2 \mathrm{~m}$ d'altitude s'est créée associée à un recul du pied de dune d'une quinzaine de mètres (fig. 7). Au nord des enrochements, l'escarpement est moins marqué, avec un recul de la dune inférieur à $10 \mathrm{~m}$ (fig.6). Un lien est observable entre la position des enrochements et la modification du profil de la plage: en effet plus on s'éloigne de la zone de stabilisation, plus le recul de la dune et l'abaissement de la plage sont négligeables (fig.8). Néanmoins la présence d'un chenal associé à un courant d'arrachement a également été observé dans la partie Sud de la plage et aurait pu contribuer à accentuer l'érosion (e.g. CASTELLE et al., 2015).

Précédemment, l'interaction négative entre les constructions en dur et l'environnement (dune et plage) ont été décrites selon trois grandes catégories : réduction de la largeur de la plage, érosion passive et érosion active (e.g. PILKEY \& WRIGHT, 1988). L’impact des enrochements sur la plage de Biscarrosse semblerait rentrer dans la dernière catégorie. En effet, à marée haute les vagues viennent taper le pied de dune libérant du sédiment, à disposition du haut de plage. La présence d'aménagements aurait tendance à bloquer ces échanges sédimentaires entre la dune et la plage, abaissant ainsi le niveau de celle-ci. La zone nord de la plage n’étant pas stabilisée, l'érosion ne résulte alors que de l'adaptation de la plage à l'accumulation des actions de chaque tempête.

Toutefois, d'autres hypothèses sont à prendre en compte concernant la variabilité longitudinale de la réponse de la plage, comme la présence d'un courant d'arrachement face aux ouvrages, ou encore le rôle des apports sédimentaires de la dérive littorale (longshore). En effet, un courant de baïne a pu être observé lors des différentes campagnes de terrain et pourrait être lié à la mise en place du "end-of-wall effect" visible de part et d'autre des enrochements (MCDOUGAL et al., 1987). Afin de mieux évaluer le rôle de chaque processus, la campagne de levés DGPS haute fréquence est maintenue jusqu’à l'été, et sera associée à l'étude d’images vidéo. 
Thème 2 - Dynamique sédimentaire

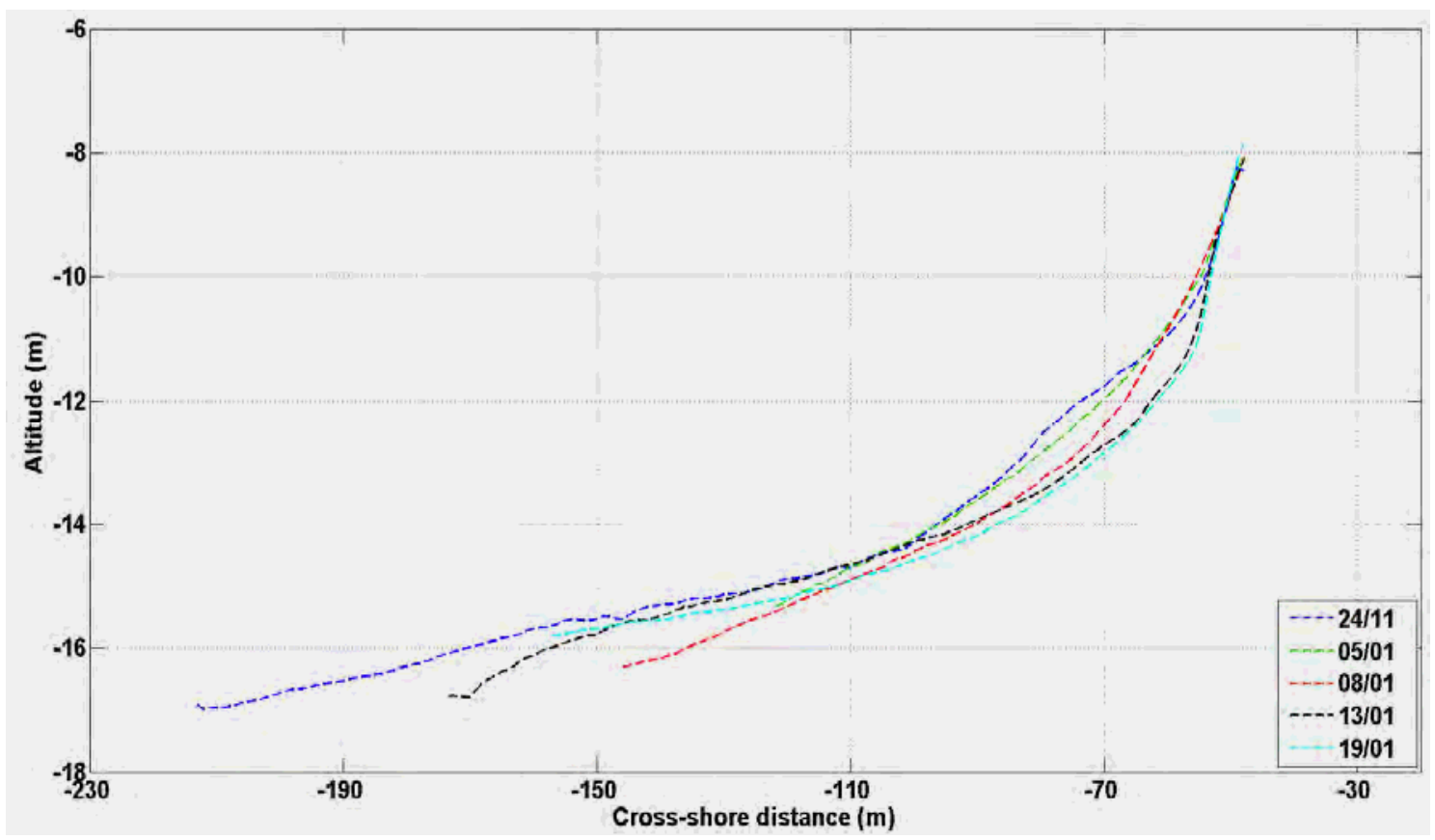

Figure 6. Profils topographiques représentant la partie au nord des enrochements. Les données sont exprimées en valeurs absolues.

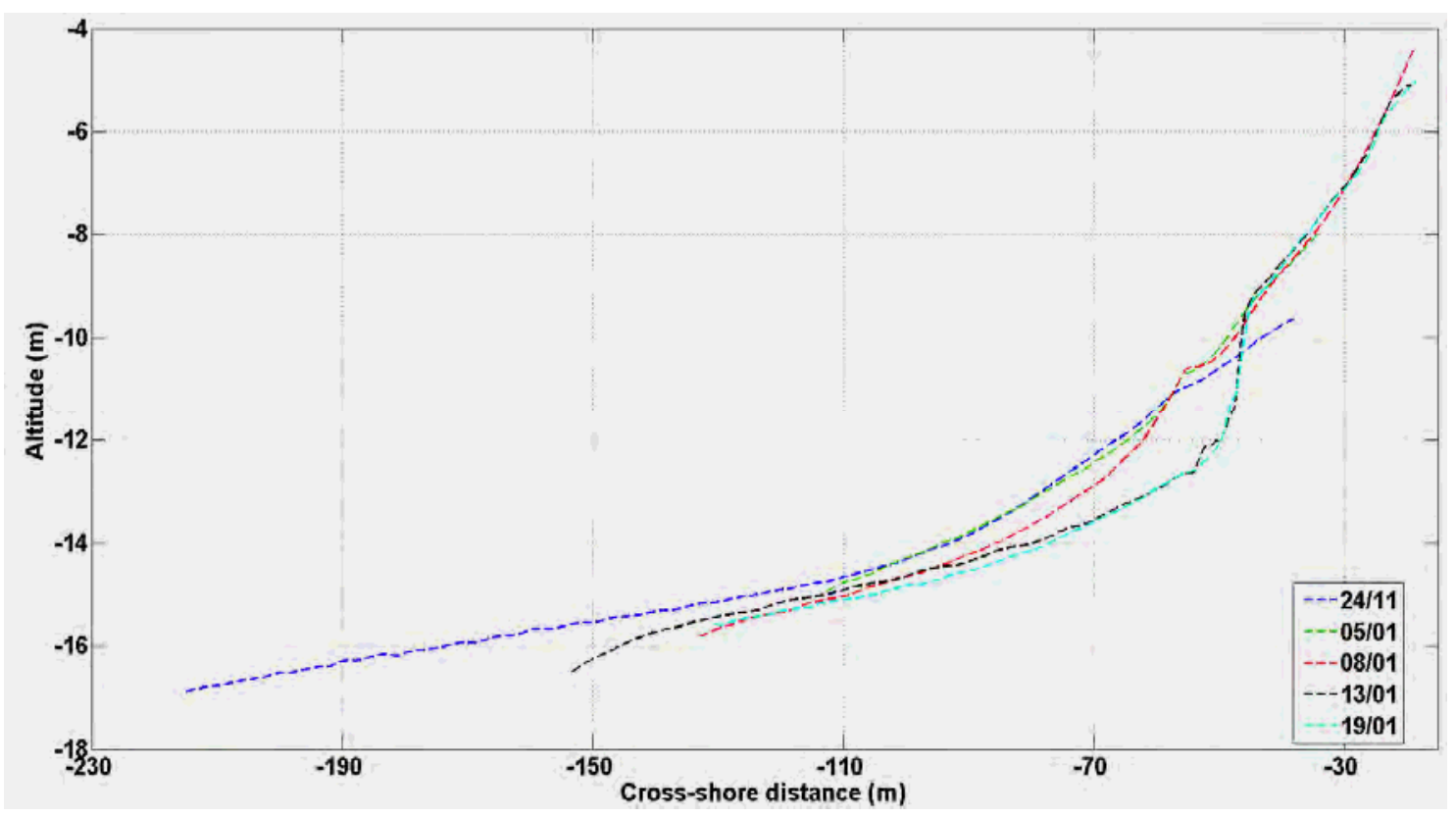

Figure 7. Profils topographiques représentant la partie au sud des enrochements. Les données sont exprimées en valeurs absolues. 


\section{XIV ${ }^{\text {èmes }}$ Journées Nationales Génie Côtier - Génie Civil \\ Toulon, 29 juin au $1^{\text {er }}$ juillet 2016}

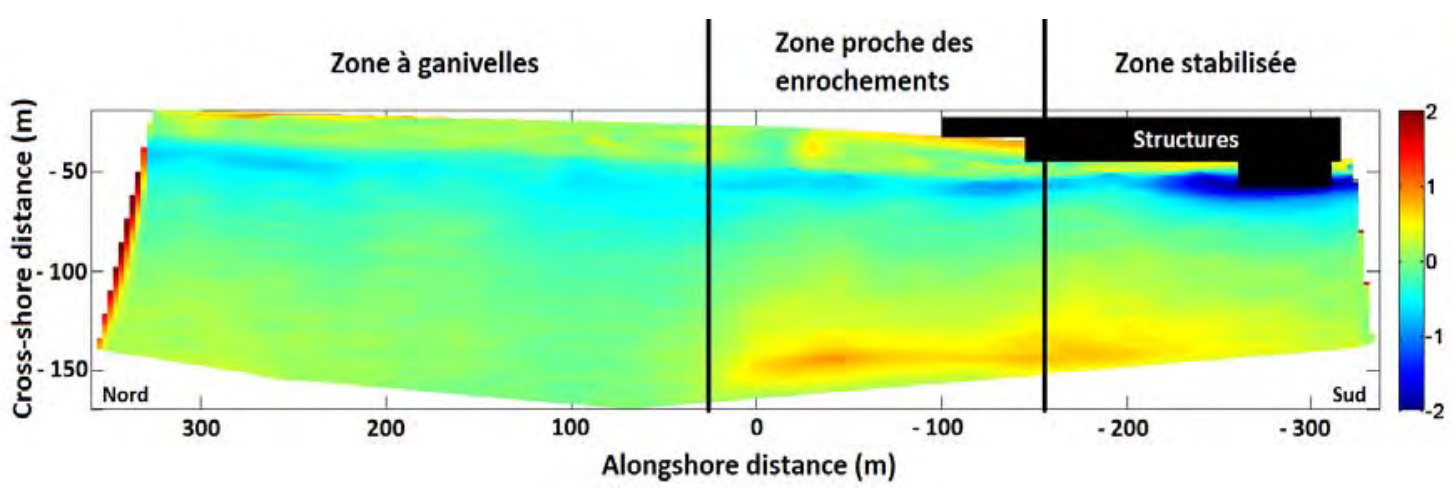

Figure 8. Carte d'évolution de Biscarrosse plage entre les 9 et 13 Janvier 2016.

\section{Conclusions}

Les levés DGPS haute fréquence sur la plage de Biscarrosse (Aquitaine-France) pendant le premier cluster de l'hiver 2015/2016 ont permis la mise en évidence d'une importante variabilité longitudinale de la réponse de la plage aux évènements de tempête. D’après les données, la 3ème tempête du cluster a été la plus effective : elle combine à la fois de forte hauteurs de vague et une marée de vives-eaux. Il apparaît qu'une première piste expliquant la différence de réponse longshore de la plage à son action soit liée aux modes de gestion du littoral biscarrossais. La présence d'enrochements pour protéger les hôtels et rares habitations construits sur la dune semble contribuer à un appauvrissement de l'alimentation du haut de plage par les dunes littorales. Cette variation longshore de la réponse du système dune/plage aux tempêtes pose maintenant la question du possible impact des enrochements sur les périodes de ré-engraissement, avec l'hypothèse proposée par DAVIS et ANDRONACO (1987) d'une augmentation du temps nécessaire à la plage pour récupérer. De nouveaux levés complétés par l'exploitation d'images vidéo permettront l'identification de tous les processus expliquant cette variabilité longitudinale (stabilisations, courants d'arrachement, position des barres inter et sub-tidales, rôle de la dérive littorale, etc..) et le rôle de chacun sur l'érosion/accrétion de la plage de Biscarrosse.

\section{Références bibliographiques}

ALMAR R., CASTELLE B., RUESSINK B.G., SENECHAL N., BONNETON P., MARIEU V. (2009). High-frequency video observation of two nearby double-barred beaches under high-energy wave forcing. J.C.R. SI56, pp 1706-1710.

BA A., SENECHAL N. (2013). Extreme winter storm versus summer storm: morphological impact on a sandy beach. J.C.R. SI, Vol. 65 (2013), pp 648-653. http://dx.doi.org/10.2112/si65.110.1

BUTEL R., DUPUIS H., BONNETON P. (2002). Spatial variability of wave conditions on the French Aquitanian coast using in-situ data. J.C.R. SI, Vol. 36, pp 96-108. 
CASTELLE B., MARIEU V., BUJAN S., et al. (2015). Impact of the winter 2013-2014 series of severe Western Europe storms on a double-barred sandy coast: Beach and dune erosion and megacusp embayments. Geomorphology, Vol. 238, pp 135-148. http://dx.doi.org/10.1016/.j.geomorph.2015.03.006

CASTELLE B., BONNETON P., DUPUIS H., SENECHAL N. (2007). Double bar beach dynamics on the high-energy meso-macrotidal French Aquitanian Coast: a review. Mar. Geol., Vol. 245, pp 141-159. http://dx.doi.org/10.1016/j.margeo.2007.06.001

DAVIS R. A., ANDRONACO M. (1987). Hurricane effects and post-storm recovery, Pinellas County, Florida (1985-1986). Coastal Sediments. ASCE, p. 1023-1036.

DOLAN R., DAVIS R.E. (1994). Coastal storm hazards. J.C.R., pp 103-114.

ELLS K., MURRAY A.B. (2012). Long-term, non-local coastline responses to local shoreline stabilization. Geophysical Research letters, Vol. 39, L19401. http://dx.doi.org/10.1029/2012gl052627

McDOUGAL W.G., STURTEVANT M.A., KOMAR P.D. (1987). Laboratory and Field Investigations of the Impact of Shoreline Stabilization Structures on Adjacent Properties. Coastal Sediments. ASCE. pp 961-973.

MICALLEF A., WILLIAMS A.T. (2002). Theoretical strategy considerations for beach management. Ocean \& Coastal management, Vol. 45, pp 261-275. http://dx.doi.org/10.1016/S0964-5691(02)00058-3

PERON C., SENECHAL N. (2011). Dynamic of a meso to macro-tidal double barred beach: inner bar response. J.C.R.,SI, Vol. 64, pp 120-124.

PILKEY O.H., WRIGHT H. L. (1988). Seawalls versus beaches. J.C.R., p. 41-64.

PILKEY O.H., HUME T.M. (2001). The shoreline erosion problem: lessons from the past. Water and Atmosphere, Vol. 9, pp 22-23.

SENECHAL N., COCO G., CASTELLE B., MARIEU V. (2015). Storm impact on the seasonal shoreline dynamics of a meso- to macrotidal open sandy beach (Biscarrosse, France). Geomorphology, Vol. 228, pp 448-461. http://dx.doi.org/10.1016/j.geomorph.2014.09.025 WRIGHT L.D., SHORT A.D. (1984). Morphodynamic variability of surf zones and beaches: a synthesis. Mar. Geol., Vol. 56, pp 93-118. http://dx.doi.org/10.1016/00253227(84)90008-2 